\title{
Duration discrimination of filled and empty auditory intervals: Cognitive and perceptual factors
}

\author{
THOMAS H. RAMMSAYER \\ University of Giessen, Giessen, Germany \\ and \\ SUSAN D. LIMA \\ University of Wisconsin, Milwaukee, Wisconsin
}

\begin{abstract}
Adult subjects were presented with two auditory stimuli per trial, and their task was to decide which of the two was longer in duration. An adaptive psychophysical procedure was used. In Experiments 1, 2, and 4, the base duration was 50 msec, whereas in Experiment 3, the base duration was 1 sec. In Experiments 1, 2, and 4, it was found that filled intervals (continuous tones) were discriminated more accurately than empty intervals (with onset and offset marked by clicks). It was concluded that this difference was perceptual rather than cognitive in nature, since performance on filled and empty intervals was not affected by increasing cognitive load in a dual-task procedure (Experiment 2) but was affected by backward masking (Experiment 4). In contrast, the results of Experiment 3 showed that duration discrimination of filled auditory intervals of longer duration was cognitively influenced, since performance was impaired by increasing cognitive load. Implications for notions of perceptual processing and timing mechanisms underlying differences in duration discrimination with filled and empty intervals are discussed.
\end{abstract}

There are two types of stimuli used in time perception studies. One type is the empty (silent) interval and the other type is the filled interval. In auditorily marked empty intervals, for example, only the onset and the offset of the interval are marked by clicks, whereas in filled intervals, a tone or noise burst is presented continuously throughout the interval. Thus, in empty intervals, there is no auditory stimulus presented during the interval itself. Surprisingly, there are very few published studies on the influence of filled versus empty intervals on performance in time perception experiments involving brief intervals (i.e., intervals less than $1 \mathrm{sec}$ in duration).

In her review article, Allan (1979) suggested that given the results of two duration-discrimination studies reported by Abel (1972a, 1972b), one can conclude that performance with filled intervals appears to be more accurate than performance with empty intervals. However, this comparison was not the focus of Abel's studies. In a study by Craig (1973), subjects had to adjust the time between two $1000-\mathrm{Hz}$ tones until it appeared equal in duration to the first tone. Craig found a constant error in the percep-

Portions of this research were presented at the annual convention of the American Psychological Association, in San Francisco, August 1991. Preparation of this article was supported by NIH Grant AG09130-01 to Susan D. Lima. We thank Lester Krueger, Lorraine Allan, and Lloyd Avant for helpful comments. Correspondence may be addressed to Thomas H. Rammsayer, Department of Psychology, University of Giessen, Otto-Behaghel Str. 10F, D-6300 Giessen, Germany (e-mail: Thoram@DGIHRZ01.bitnet). tion of empty intervals relative to filled intervals. This finding joins those of Abel (1972a, 1972b) in suggesting that performance on filled intervals is more accurate than performance on empty intervals. Although Craig concluded that different timing mechanisms may be required for processing filled and empty intervals, he did not propose any specific mechanisms. Thus, as Allan (1979) stated, no theory has been proposed to explain why it should be the case that filled intervals are discriminated better than empty intervals.

Our major focus was on very brief auditory intervals, which we define as intervals of less than $100 \mathrm{msec}$ in duration. The base duration was $50 \mathrm{msec}$ in Experiments 1,2 , and 4 . The goal of the first experiment was to directly establish the direction and degree of performance differences in duration discrimination of filled versus empty intervals. The purpose of the remaining three experiments was to elucidate the bases of any observed differences between filled and empty intervals. Experiments 2 and 3 addressed the issues of whether duration discrimination is cognitively mediated and whether differences in performance between filled and empty intervals are due to differential cognitive loads in the two conditions. Another goal of Experiment 3 was to investigate cognitive influences in duration discrimination of longer intervals, those of approximately $1 \mathrm{sec}$ in duration. In Experiment 4, backward masking was used to test the view that duration discrimination of very brief auditory intervals is based primarily on processing at a perceptual level. Thus, Experiment 4 was intended to provide results rele- 
vant to the view that differences in performance between filled and empty intervals are due to differences in perceptual processing.

\section{EXPERIMENT 1}

In Experiment 1, subjects were presented with two consecutive intervals per trial, and their task was to decide which of the two intervals was longer in duration. As a measure of discrimination performance, difference thresholds were computed. We chose not to use traditional psychophysical methods such as the method of constant stimuli and instead used an adaptive procedure, the transformed up-down procedure described by Levitt (1971). Fixed methods suffer from the drawback that subjects are often confronted with stimuli far removed from the desired level of the independent variable. For such stimuli, the proportion of positive responses is either near zero or near unity; that is, little information is gained from these trials, and hence the fixed methods are relatively inefficient. A general advantage of adaptive procedures is their higher efficiency and greater flexibility, resulting in fast and reliable estimation of the value of a stimulus variable that yields a threshold response.

\section{Method}

Subjects. The subjects were 12 male and 12 female adults ranging in age from 20 to 58 years $(M=32.3, S D=10.2)$. All subjects had normal hearing.

Apparatus and Stimuli. The presentation of the intervals and the recording of the subjects' responses was controlled by an IBMAT-compatible computer. Auditory tones were generated by a computer-controlled sound generator. The frequency of the tone used in the filled intervals was $1000 \mathrm{~Hz}$, and the intensity was $67 \mathrm{~dB}$. The empty intervals were marked by onset and offset clicks $3 \mathrm{msec}$ in duration, with an intensity of $88 \mathrm{~dB}$. This higher physical intensity was necessary to achieve equal loudness in the two conditions. These intensity levels were chosen on the basis of the results of a prior pilot experiment in which 12 subjects were asked to adjust the loudness of a 3-msec click until it matched that of a 50 -msec tone.

Procedure. The stimuli were presented through headphones (Vivanco Model SR85). An experimental session consisted of one block of empty and one block of filled intervals; the order of blocks was counterbalanced across subjects. Each block consisted of 50 trials, and each trial consisted of two stimuli, one 50 -msec standard interval, and one comparison interval. The comparison interval varied in duration from trial to trial, depending on the subject's previous responses according to the transformed up-down procedure described by Levitt (1971), which converges on a probability of hits of $70.7 \%$. Some advantages of this procedure over traditional psychophysical methods are its robustness, small-sample reliability, and avoidance of floor and ceiling effects. The duration of the comparison interval changed with a constant step size of $8 \mathrm{msec}$ for Trials 1-10, 4 msec for Trials 11-30, and 2 msec for Trials 31-50. The initial value of the comparison interval was $98 \mathrm{msec}$. The order of presentation for the standard interval and the comparison interval was randomized and balanced, with each interval being presented first in $50 \%$ of the trials. The subjects were not informed that there was a constant standard interval of $50 \mathrm{msec}$ in every trial. When asked after the experiment if they had been aware of different presentation orders of a constant standard and a variable comparison interval, they reported that they had not.
Each subject was seated at a table with a keyboard and a computer monitor in a sound-attenuated room. To initiate a trial, the subject pressed the space bar; the auditory presentation began 900 msec later. The two intervals were presented with an interstimulus interval of $900 \mathrm{msec}$. The subject's task was to decide which of the two intervals was longer and to indicate his or her decision by pressing one of two designated keys on the keyboard; one key was labeled "first interval longer" and the other was labeled "second interval longer." The instructions to the subjects emphasized accuracy; there was no requirement to respond quickly. After each response, visual feedback ("CORRECT" or "FALSE") was displayed. The next trial started when the subject pressed the space bar again.

The experimental trials were preceded by practice trials, in which the adaptive procedure was also used. The purpose of the practice trials was to ensure that the subjects understood the instructions and to familiarize them with the stimuli. A subject began the experimental trials only after having achieved six consecutive correct responses in the practice trials. Despite the fact that the starting value of the comparison interval was $98 \mathrm{msec}$, the number of practice trials varied from subject to subject. This occurred primarily because different subjects required different numbers of trials to become accustomed to processing the empty intervals.

As a measure of performance, mean differences between standard intervals and comparison intervals were computed for the last 20 trials within each block. This measure represents an estimate of the individual $70.7 \%$ difference threshold in milliseconds in relation to a standard interval of $50 \mathrm{msec}$. Thus, better performance on duration discrimination is indicated by smaller values.

\section{Results}

A $t$ test revealed that duration discrimination was significantly better with filled intervals than with empty intervals; the respective mean $70.7 \%$ difference thresholds were $6.6 \mathrm{msec}$ and $20.9 \mathrm{msec}[t(23)=5.81, p<.001]$. Clearly, performance on filled intervals was superior to performance on empty intervals. The results of Experiment 1 established that subjects were able to discriminate filled intervals differing from each other by approximately $7 \mathrm{msec}$, but could only discriminate empty intervals if they differed from each other by at least $21 \mathrm{msec}$.

\section{Discussion}

At least two perceptual processes can account for the finding that filled intervals are discriminated with finer temporal resolution than empty intervals. One of these processes is sensory integration. Sensory integration allows subjects to base their responses on available nontemporal as well as temporal cues. The major nontemporal cue that is present in filled intervals but not empty intervals is physical energy. In filled intervals, for example, physical energy increases as the duration of the interval increases, but in empty intervals, physical energy is constant (Carbotte \& Kristofferson, 1971, 1973); in fact, there is virtually no physical energy present. Thus, subjects should perform better with filled intervals if duration discriminations are based on loudness rather than on temporal information. That is, true temporal information would not be used, because longer filled durations would be judged to be longer simply because they sounded louder than shorter filled durations. However, there is 
convincing evidence that such a possible confound or artifact can be ruled out and furthermore that discriminability of short auditory durations is independent of energy values. In a series of experiments done with a signal detection paradigm, Creelman (1962) studied the effects of stimulus intensity on duration discrimination with filled auditory intervals in the millisecond range. Performance in discriminating between a 100- and a 130-msec interval increased with stimulus intensity, but only at low signal-to-noise ratios. When the intervals were made clearly audible, this effect leveled off and discrimination performance became independent of stimulus intensity. Furthermore, on the basis of his results with filled auditory intervals ranging from $\mathbf{4 0}$ to $\mathbf{6 4 0} \mathrm{msec}$, Creelman (1962) concluded that "duration discrimination depends on sufficient intensity to mark the time unambiguously; it depends on detectability but not on loudness" (p. 592). Similarly, Abel (1972b) found that discrimination of tone bursts with base durations of 5,40 , and $320 \mathrm{msec}$ proved to be independent of the intensity of the stimulus ( $85 \mathrm{vs.}$ $65 \mathrm{~dB}$ ). In a study of empty auditory intervals ranging in duration from 50 to $250 \mathrm{msec}$, Carbotte and Kristofferson (1973) employed changes in marker intensity of $37 \mathrm{~dB}$ and found no evidence that duration discrimination was based on energy-dependent cues. Taken together, these results suggest that duration discrimination of both filled and empty auditory intervals in the range of milliseconds is independent of signal energy. (See Allan, 1979, and Allan \& Kristofferson, 1974, for reviews.)

The second perceptual process that may be at work is masking; that is, smaller difference thresholds with filled as opposed to empty intervals may be partially due to the existence of forward or backward masking effects in the empty intervals, with one click partially masking the other. There should be no appreciable masking effects in the filled-interval comparisons, since the second interval within a trial was presented $900 \mathrm{msec}$ after the offset of the first interval. It has been shown that masking is not effective beyond approximately $200-250 \mathrm{msec}$ (see, e.g., Deatherage \& Evans, 1969; Massaro, 1972).

An alternative to the perceptual view is the view that duration discrimination is cognitively mediated, and that discriminating empty intervals is cognitively more complex than discriminating filled intervals. Within a cognitive framework, the result could be explained as arising from the necessity of processing more events in emptyinterval comparisons as opposed to filled-interval comparisons; our empty-interval comparisons required the processing of four events, whereas filled-interval comparisons required the processing of only two events.

\section{EXPERIMENT 2}

At this point, it appears that either a perceptual explanation or a cognitive explanation may account for the results of Experiment 1. The purpose of Experiments 2 and 3 was to test predictions of the cognitive explanation. These predictions were inspired by studies that have shown that increasing the amount of stimulus information within an interval while duration is held constant can strongly influence subjects' judgments of duration. Three major theories of these findings have emerged: the storage size model (Block, 1974; Mulligan \& Schiffman, 1979; Ornstein, 1969), the processing effort model (Avant, Lyman, \& Antes, 1975; Fraisse, 1979; Thomas \& Weaver, 1975), and the change model (Block \& Reed, 1978; Poynter \& Homa, 1983). All these models posit that duration judgment is cognitively mediated.

If duration discrimination of very brief auditory intervals is cognitively mediated, we would predict that duration discrimination under relatively high cognitive load would be more difficult than duration discrimination under lower cognitive load. To increase cognitive load, we used a dual-task procedure, in which the primary task was duration discrimination and the secondary task was an obviously cognitive task, word learning. Results from the dual-task conditions were compared with results from single-task conditions identical to the two conditions of Experiment 1. The rationale for this procedure was the assumption that if two tasks compete for the same pool of cognitive processing effort, then having to perform both tasks simultaneously should impair performance relative to when either task is performed alone (see, e.g., Baddeley, 1981; Baddeley \& Hitch, 1974). Furthermore, increased cognitive load should affect discrimination of empty intervals more adversely than discrimination of filled intervals. Such a result would be predicted on the view that empty intervals are more difficult to process than filled intervals because empty intervals (consisting of four events) require more cognitive effort than filled intervals (consisting of two events).

\section{Method}

Subjects. There were four experimental conditions and one additional control condition in this experiment. The subjects in the experimental conditions were 14 female and 10 male students ranging in age from 20 to 29 years $(M=23.8, S D=3.1)$. All of these subjects had normal hearing. The subjects in the control condition were 6 female and 6 male students ranging in age from 21 to 35 years $(M=27.3, S D=4.8)$. All subjects were from the University of Giessen, and none participated in any of the other experiments.

Apparatus. The apparatus was the same as in Experiment 1.

Design and Stimuli. Two independent variables-type of interval and cognitive load-were factorially combined in a within-subject design to yield four experimental conditions. Type of interval was manipulated as in Experiment 1. The two levels of cognitive load were high and low, with the low-cognitive-load conditions being identical to the conditions used in Experiment 1. High cognitive load was induced by requiring subjects to learn visually presented words as a secondary task. The resulting four experimental conditions were low load, filled interval; low load, empty interval; high load, filled interval; and high load, empty interval.

Order of conditions was counterbalanced across subjects. However, in order to avoid duplication of words in the two highload conditions, each subject was presented with Word List 1 (nouns) in one of his or her high-load conditions and Word List 2 (verbs) in the other. Word list was counterbalanced across the emptyand filled-interval conditions. Each word list consisted of $25 \mathrm{Ger}$ man words, shown in the Appendix along with their English trans- 
lations. The two word lists were approximately matched on mean word length, word frequency, and difficulty of recall (on the basis of pilot work).

To test word recall in the absence of the duration-discrimination task, a control condition was run in which a different group of subjects performed the word-learning task only. Half of these subjects received the noun list before the verb list, whereas the other half received the lists in the reverse order. The only dependent variable for the control condition was recall rate of nouns and verbs.

Procedure. The procedure for the two low-load conditions was identical to that of Experiment 1. For the high-load conditions, a secondary task of word learning was added to the primary task of duration discrimination. In these conditions, the sequence of events was as follows: 500 msec after the subject started a trial by pressing the space bar, one word appeared in the center of the monitor screen and remained on for $1,500 \mathrm{msec}$. After a delay of $900 \mathrm{msec}$, the first auditory interval was presented, followed $900 \mathrm{msec}$ later by the second auditory interval. The subject was instructed to memorize the word for a later recall test, perform the durationdiscrimination task, and indicate his or her response by pressing the appropriate key. The instructions emphasized the importance of accuracy in both tasks. Each subject was presented with the words in a different random order, and each subject saw each word twice, both times within the same condition. No word was repeated until all the words had been presented once. A word-recall test occurred at the end of each high-load condition.

In the control condition, words were visually presented at exactly the same rate as in the experimental conditions, but no auditory stimuli were presented, so that no duration discriminations were performed. In the high-load experimental conditions and in the control condition, after all the trials had been completed, the subjects were asked to write down all the words they remembered and were given a maximum of $3 \mathrm{~min}$ to do so. No subject required the entire $3 \mathrm{~min}$, however.

\section{Results}

Experimental conditions. Results from the durationdiscrimination task were computed as in Experiment 1. An additional dependent variable in this experiment was the number of words recalled correctly (in the high-load conditions). In $t$ tests, it was shown that there was no statistically significant effect of word list (nouns vs. verbs) on duration-discrimination performance on either filled $(p=.20)$ or empty intervals $(p=.62)$. Also, there was no statistically significant difference in recall performance for nouns as opposed to verbs ( $p=.15$ for filled intervals, $p=.92$ for empty intervals). Because type of word list had no significant effects, we collapsed across word lists in subsequent analyses.

The duration-discrimination difference threshold data are presented in Table 1. A two-way analysis of variance revealed a statistically significant main effect of type of interval $[F(1,23)=29.35, p<.001]$; the mean threshold value was $18.8 \mathrm{msec}$ for the empty intervals and $7.1 \mathrm{msec}$

Table 1

70.7\% Difference Thresholds, Experiment 2 (in Milliseconds)

\begin{tabular}{ccc}
\hline & \multicolumn{2}{c}{ Cognitive Load } \\
\cline { 2 - 3 } Type of Interval & Low & High \\
\hline Filled & 7.3 & 7.0 \\
Empty & 18.7 & 18.9 \\
\hline
\end{tabular}

for the filled intervals. There was, however, no significant main effect of cognitive load; threshold values in the low-load conditions averaged $13.0 \mathrm{msec}$, compared to $12.9 \mathrm{msec}$ in the high-load conditions. In addition, cognitive load did not interact with type of interval. Therefore, the predictions from the cognitive framework were not borne out by the data. Duration discrimination was not more difficult under high cognitive load than under low cognitive load, and because increasing cognitive load had virtually no effect on duration-discrimination performance, empty intervals were not affected more by cognitive load than were filled intervals.

There was no significant effect of type of interval on word-recall performance ( $p=.86$ ); recall performance averaged $47.4 \%$ in the high-load, empty-interval condition and $47.8 \%$ in the high-load, filled-interval condition. Therefore, just as the word-recall task did not affect duration-discrimination performance, the type of durationdiscrimination task did not affect word-recall performance. Once again, there was no support for the notion that duration discrimination is cognitively mediated or that temporal processing of empty intervals requires more cognitive effort than does processing of filled intervals.

Control condition. The subjects in the control condition recalled an average of $75.0 \%$ of the verbs and an average of $75.0 \%$ of the nouns. Because type of word list had no effect on word-recall rate, we collapsed across this variable in the subsequent analysis. The recall rate of $75.0 \%$ was significantly greater than the $47.6 \%$ mean rate observed in the two high-load conditions $[t(34)=5.58$, $p<.001]$.

\section{Discussion}

The results of Experiment 2, like those of Experiment 1, showed that empty intervals were more difficult to discriminate than filled intervals. However, the fact that durationdiscrimination performance on filled and empty intervals did not interact with cognitive load, and the fact that the same word-recall rate was observed in both the high-load, empty-interval condition and the high-load, filled-interval condition, clearly argues against the view that the difference in duration-discrimination performance on filled and empty intervals was due to cognitive mediation.

At first glance it may appear that the superior wordrecall rate observed in the control condition relative to the high-load conditions poses a problem for the view that duration discrimination of very brief intervals is not cognitively based. If duration discrimination in this range is purely a perceptual process, why should it be the case that subjects not required to perform duration discriminations recalled more words than those required to perform duration discriminations? We would argue that the timing mechanism underlying duration discrimination in the millisecond range is perceptual in nature, but that the task demands of the decision stage of any discrimination task do consume cognitive capacity. This view is the one that can best explain the finding that duration-discrimination per- 
formance was unimpaired by the presence of the wordlearning task, whereas performance on the word-learning task was impaired by the presence of the durationdiscrimination task. According to this view, subjects in the control condition recalled more words than subjects in the high-load experimental conditions because, unlike the experimental-condition subjects, the control-condition subjects did not have to make any decisions and therefore could devote more cognitive capacity to word encoding and rehearsal. If subjects regarded the duration-discrimination judgment as primary and the word-learning task as secondary, then duration-discrimination judgments would be less impaired by word learning than word-learning performance would be by duration discrimination.

The critical point, however, is that cognitive-processing differences do not exist between filled and empty auditory intervals. Had it been the case that differences in duration-discrimination performance between filled and empty intervals were due to cognitive processing, the high-load conditions would have been more difficult than the low-load conditions, and the empty intervals would have been more adversely affected by high cognitive load than would the filled intervals. Because there were no such findings in Experiment 2, it appears that cognitive involvement in duration-discrimination judgment is restricted to the decision stage and that the timing mechanism underlying duration discrimination in the millisecond range is perceptual in nature.

\section{EXPERIMENT 3}

Our lack of evidence for a cognitive timing mechanism for empty and filled intervals (ranging from 50 to $98 \mathrm{msec}$ ) in Experiments 1 and 2 stands in agreement with the view espoused by Michon (1985), who stated that "below $0.5 \mathrm{sec}$ information processing is of a highly perceptual nature, fast, parallel and not accessible to cognitive control"' (p. 40). If, as Michon's view implies, duration discrimination of auditory intervals greater than $0.5 \mathrm{sec}$ in duration is cognitively mediated, we should be able to demonstrate a deleterious effect of increasing cognitive load on duration discrimination of intervals in the range of seconds. Such a demonstration was the goal of Experiment 3 , in which we applied a technique similar to that of Experiment 2, except that longer auditory intervals were studied. The duration of the standard interval in Experiment 3 was $1 \mathrm{sec}$.

\section{Method}

Subjects. The subjects were 7 male and 9 female students at the University of Giessen ranging in age from 21 to 41 years $(M=$ $25.4, S D=8.2$ ). All subjects had normal hearing and had not participated in any of the other experiments.

Apparatus. The apparatus was the same as in Experiments 1 and 2.

Design and Stimuli. The design was similar to that of Experiment 2 except that there were only two conditions, a low-load, filledinterval condition and a high-load, filled-interval condition. In Experiment 3 , the standard interval was $1,000 \mathrm{msec}$ and the initial value of the comparison interval was $2,000 \mathrm{msec}$. Duration of the comparison interval changed with a constant step size of $200 \mathrm{msec}$ for Trials $1-10,100 \mathrm{msec}$ for Trials 11-30, and $50 \mathrm{msec}$ for Trials 31-50. Empty intervals were excluded because pilot work with 8 subjects revealed that no subject could readily perceive the pattern of four clicks as two consecutive empty intervals. Because recall of nouns and verbs had been virtually identical in Experiment 2 , only nouns were used in Experiment 3. Order of conditions (low load, high load) was counterbalanced across subjects.

Procedure. The procedure was identical to those of the low-load, filled-interval and high-load, filled-interval conditions of Experiment 2, except that only nouns were used, and the standard and comparison intervals were longer in duration, as noted above.

\section{Results}

Duration discrimination was significantly better in the low-load condition than in the high-load condition; the respective mean $70.7 \%$ difference thresholds were 106.6 and $152.7 \mathrm{msec}[t(15)=2.57, p=.02]$. Thus, it is obvious that duration discrimination in the range of seconds was impaired by an increase in cognitive load. In the highload condition, the word-recall rate was $54.8 \%$, which was not significantly different from the $47.6 \%$ recall rate observed in the filled-interval conditions of Experiment 2 $(p=.48)$. The $54.8 \%$ recall rate was significantly less than the $75.0 \%$ recall rate observed in the control condition of Experiment $2[t(26)=3.75, p=.001]$.

\section{Discussion}

Unlike Experiment 2, Experiment 3 yielded a pronounced impairment of duration-discrimination performance, which was due to increased cognitive load. Taken together, the findings of these two experiments indicate that the timing mechanism underlying duration discrimination of filled auditory intervals in the range of seconds is dependent on cognitive processing, whereas the timing mechanism underlying duration discrimination of intervals in the range of milliseconds is more likely to be dependent on perceptual processing. These results agree with Michon's (1985) view that intervals greater than $500 \mathrm{msec}$ in duration are more likely to be cognitively processed than briefer intervals. Some additional evidence converging on this conclusion comes from pharmacopsychological studies of time perception (Mitrani, Shekerdjiiski, Gourevitch, \& Yanev, 1977; Rammsayer, Vogel, Serota, \& Gottheil, 1989) and from a study of sensory compensation in blind subjects performing auditory discriminations (Rammsayer, 1991).

The outcome of Experiment 3 also provides convincing evidence that our dual-task procedure was sufficiently sensitive to detect cognitive involvement in timing mechanisms, and thus lends support to our interpretation of the results of Experiment 2. It is interesting that recently, Fortin and Rousseau (1987) have also fruitfully applied a dualtask procedure to investigate the interaction between time estimation and cognitive demands.

\section{EXPERIMENT 4}

If, as we have argued, duration discrimination in the range of milliseconds is based on perceptual processing, it should be the case that auditory masking will have a 
profound effect on performance. Masking seems to be an appropriate technique for studying stimulus processing at the perceptual level, since the locus of masking is prior to entry into short-term memory (see, e.g., Shiffrin, 1976). A substantial body of evidence indicates that both forward masking and backward masking markedly impair duration-discrimination performance on filled auditory intervals in the range of approximately $40-90 \mathrm{msec}$ (e.g., Allan \& Rousseau, 1977; Kallman, Beckstead, \& Cameron, 1988; Kallman, Hirtle, \& Davidson, 1986; Kallman \& Morris, 1984). Although the effect of masking on duration discrimination of filled auditory intervals has been established, to our knowledge no studies have been performed to compare the effect of masking on filled as opposed to empty auditory intervals.

In Experiment 4, a backward masking paradigm was used with filled and empty auditory intervals of the same very brief duration as those used in Experiments 1 and 2. Because the effects of backward masking can be regarded as independent of the capacity limitations of short-term memory (see, e.g., Hawkins \& Presson, 1977; Massaro, 1976), any effects of backward masking that we observe can be attributed to perceptual processing. Thus, if duration-discrimination performance is impaired by backward masking, this would constitute supporting evidence that duration-discrimination judgments of very brief empty as well as filled auditory intervals are based on processing at the perceptual level, and this finding in conjunction with the results of Experiment 2 would argue against the view that the difference in discrimination performance between empty and filled intervals in the range of milliseconds is due to cognitive-processing differences. The specific pattern of results may also illuminate the reason underlying the superiority of duration-discrimination performance with filled as opposed to empty intervals.

\section{Method}

Subjects. The subjects were 12 female and 12 male students at the University of Giessen ranging in age from 20 to 38 years $(M$ $=24.3, S D=4.0$ ). All subjects had normal hearing and had not participated in any of the other experiments.

Apparatus. The apparatus was the same as in Experiments 1, 2 , and 3 .

Design and Stimuli. Two independent variables-type of interval and masking/no masking-were factorially combined in a withinsubject design. The two no-masking conditions were identical to the two conditions of Experiment 1. In the masking conditions, the first and second interval within a trial were each followed by a 200msec masking tone of $328 \mathrm{~Hz}$ at an intensity of $73 \mathrm{~dB}$. (Recall that the intensity of the filled intervals was $67 \mathrm{~dB}$ and that of the empty interval markers was $88 \mathrm{~dB}$.) The delay between the offset of the interval and the onset of the mask was $10 \mathrm{msec}$. These values were chosen to maximize any effects of the backward masking manipulation (Deatherage \& Evans, 1969; Massaro, 1972; Massaro, Cohen, \& Idson, 1976): the mask was of longer duration, different frequency, and greater subjective loudness than were the stimuli representing the intervals (although of less physical intensity than the clicks marking the empty intervals), and the mask followed each interval very closely in time. The four conditions in the design were empty interval, no masking; empty interval, masking; filled interval, no masking; and filled interval, masking. Order of conditions was counterbalanced across subjects.
Procedure. The procedure was similar to that of Experiment 1, with the exception that there were four practice blocks before the experimental blocks, one practice block corresponding to each type of experimental block. All subjects received all their practice before beginning any experimental trials. Order of practice blocks was counterbalanced across subjects. As in Experiment 1, each practice block was terminated when the subject had correctly judged six consecutive practice trials. For the empty-interval masking condition, subjects were instructed to base their judgments on the intervals each marked by two clicks. For the filled-interval masking condition, subjects were told that the masking tone was lower in frequency than the interval tone.

\section{Results}

Duration-discrimination threshold values were computed as in Experiment 1. The threshold values can be seen in Table 2. An analysis of variance confirmed that performance with masked intervals was significantly worse than performance with unmasked intervals $[F(1,23)$ $=25.95, p<.001]$; the mean duration-discrimination threshold value was $33.4 \mathrm{msec}$ for masked intervals and $16.2 \mathrm{msec}$ for unmasked intervals. Also, empty intervals were judged less accurately than filled intervals $[F(1,23)$ $=28.49, p<.001]$; the mean for empty intervals was $32.9 \mathrm{msec}$, and the mean for filled intervals was $16.7 \mathrm{msec}$. In addition, there was a statistically significant interaction between type of interval and masking/no masking $[F(1,23)=11.77, p<.002]$. Specifically, this interaction resulted because the effect of masking was greater for empty $(26.3 \mathrm{msec})$ than for filled intervals $(8.1 \mathrm{msec})$.

We also computed percent-change scores to compare the proportional increase in duration-discrimination thresholds as a result of masking. To do this, each subject's mean threshold in the unmasked condition was subtracted from the mean in the corresponding masked condition and then expressed as a percent change against the unmasked condition; thus, each subject yielded two scores, one for the filled intervals and one for the empty intervals. The mean percent-change scores were $68.9 \%$ for the filled intervals and $156.7 \%$ for the empty intervals, indicating that masking-induced impairment of empty intervals was proportionally greater than that of filled intervals $[t(23)=2.28, p=.03]$. Thus, masking impaired processing of empty intervals more than processing of filled intervals, both absolutely and proportionally.

\section{Discussion}

We observed impaired performance in duration discrimination that was due to masking in both filled and empty intervals, as would be expected if duration discrimination performance with both types of intervals were

Table 2

70.7\% Difference Thresholds, Experiment 4 (in Milliseconds)

\begin{tabular}{ccc} 
& \multicolumn{2}{c}{ Masking Condition } \\
\cline { 2 - 3 } Type of Interval & No Masking & Masking \\
\hline Filled & 12.6 & 20.7 \\
Empty & 19.7 & 46.0 \\
\hline
\end{tabular}


primarily based on processing at the perceptual level. However, we found that performance on empty intervals was much more impaired by masking than was performance on filled intervals; in fact, the absolute level of impairment in the empty-interval condition was 3.25 times greater than that in the filled-interval condition, and the proportional level of impairment was 2.27 times greater in the empty-interval condition than in the filled-interval condition.

There are several possible explanations of the greater impairment with empty intervals. First, given the assumption that backward masking overwrites the contents of the short-term auditory store (Massaro, 1972), it would be expected that backward masking would be most effective in a case in which processing of auditory information is not yet complete. Because the masking stimulus was presented $10 \mathrm{msec}$ after the offset of each of the intervals, information that had been processed within that 10 msec period should be unaffected by the mask. The finding that the mask was more effective in empty intervals than in filled intervals may indicate that processing at the time of mask onset was less complete in the case of empty intervals than in the case of filled intervals. The number of sensory events within the empty intervals was twice that within the filled intervals, which may have resulted in more time being required to complete perceptual processing of duration information in the case of the empty intervals. According to this argument, processing was interrupted at a less complete point in the empty intervals than it was in the filled intervals, resulting in particularly impaired performance on duration discrimination with empty intervals.

However, one problem with this "interruption" interpretation is that Kallman and his colleagues (Kallman et al., 1988; Kallman et al., 1986) have found that forward masking and backward masking exert equivalent effects on filled auditory durations, which led them to conclude that an interruption theory of backward duration masking is not likely to be correct. Furthermore, Kallman et al. (1988), studying the effects of ipsilateral and contralateral forward and backward masking with varying interstimulus intervals (ISIs) between target and mask, found marked increases in percentages correct under every combination of mask ear and mask position due to increases in ISI. The authors concluded that auditory-duration masking is the result of central rather than peripheral processing. They attributed their results to a "central timekeeper" (p. 35) and hypothesized that both forward and backward masking may cause a misassignment of neural pulses generated by an internal pacemaker. It can plausibly be assumed that empty intervals are more prone to misassignment than filled intervals. It should be noted, however, that Kallman and his colleagues did not study empty auditory intervals, so it has not yet been demonstrated that forward and backward masking exert equivalent effects on duration judgments of empty intervals.
Therefore, we cannot be certain that Kallman's hypothesis can be used to explain the differences we found between filled and empty auditory intervals.

\section{GENERAL DISCUSSION}

The results of Experiments 1, 2, and 4 clearly demonstrate that filled intervals are more easily discriminated than empty intervals. The pattern of results is remarkably consistent across these experiments; the overall mean difference thresholds (taking into account data from Experiment 1, Experiment 2, and the no-masking conditions of Experiment 4) were $8.8 \mathrm{msec}$ for filled auditory intervals and $19.8 \mathrm{msec}$ for empty intervals. Thus, it was demonstrated that when the intervals were filled (i.e., marked by a continuous tone), subjects could reliably discriminate the durational difference between a $50-\mathrm{msec}$ standard interval and a 58.8-msec comparison interval. In contrast, when the intervals were empty (i.e., had onset and offset marked by clicks), the smallest difference that could be reliably detected was that between the 50msec standard interval and a 69.8-msec comparison interval. Thus, difference thresholds for empty intervals were approximately $\mathbf{2 . 2 5}$ times greater than those for filled intervals.

The results of Experiment 2, in which a dual-task procedure was used to induce a higher cognitive load, argue against the view that duration-discrimination performance on very brief auditory stimuli is cognitively mediated. If performance had been cognitively mediated, it should have been the case that duration discriminations under high cognitive load would yield larger difference thresholds than would duration discriminations under low cognitive load. Instead, we found in Experiment 2 that durationdiscrimination performance was unaffected by cognitive load. Another prediction of the cognitive view was that empty intervals should be more adversely affected by $\operatorname{cog}$ nitive load than would filled intervals, and this prediction was also contradicted by the data. The likely conclusion is that duration discrimination of intervals in the range of 50-100 msec is based on processing at the perceptual level. Furthermore, the processing of empty intervals does not appear to be more cognitively complex than the processing of filled intervals, despite the fact that an emptyinterval trial involved four auditory events, whereas a filled-interval trial involved only two auditory events.

In contrast to Experiment 2, which involved intervals in the range of milliseconds, Experiment 3 demonstrated that duration-discrimination performance on longer filled intervals (approximately $1 \mathrm{sec}$ in duration) was impaired by the imposition of a high cognitive load. These findings point to the conclusion that the timing mechanism underlying duration discrimination of intervals in the range of seconds is influenced by cognitive processing, whereas the timing mechanism underlying duration discrimination of intervals in the range of milliseconds is 
uninfluenced by cognitive processing, being highly perceptual in nature.

Further evidence that duration discrimination in the range of milliseconds is based on processing at the perceptual level was provided by the results of Experiment 4, which showed that auditory backward masking had profound effects on performance. Backward masking impaired duration discrimination of both filled and empty intervals, but performance on empty intervals was much more impaired by masking than was performance on filled intervals. Thus, we demonstrated not only that empty intervals are more difficult to process than filled intervals, but also that backward masking increases the size of the difference. We now turn our attention to possible explanations of the differences in processing filled and empty intervals based on notions of perceptual processing.

One possibility is a sensory-integration explanation. According to this view, subjects' responses in durationdiscrimination tasks may be facilitated by the availability of a nontemporal cue such as the presence of a discernible physical stimulus during the interval. Performance on duration discrimination is often interpreted by the assumption of a neural counting mechanism (see, e.g., Church, 1984; Creelman, 1962; Treisman, 1963). This means that a neural pacemaker of some kind generates pulses and that the number of pulses relating to a physical time interval is the subjective representation of the duration of this interval. The higher the rate of pulses, the better the temporal resolution of the mechanism. Thus, the physiological basis of better performance on filled than on empty intervals can be envisioned as an increase in neural firing rate due to the presence of a perceivable physical stimulus in the case of filled intervals (see, e.g., Evans, 1975). This higher firing rate will result in finer temporal resolution and thus less uncertainty about interval duration in filled intervals than in empty intervals. In masked trials, if filled intervals benefit from sensory integration, at the time of the onset of the masking stimulus, processing is less complete in empty intervals than it is in filled intervals, and this results in less impairment due to masking in the filled intervals. Such a view of sensory integration does not, however, necessarily imply that an increase in stimulus intensity of the tone in the filled interval will result in noticeable improvement in durationdiscrimination performance, since it may be the case that the increase in neural firing rate in a filled-interval trial as opposed to an empty-interval trial is far greater than any differences that may result from changes in stimulus intensity within filled intervals. In fact, Creelman (1962) showed that in a duration-discrimination task with filled intervals, as long as auditory stimuli were clearly audible, changing the intensity of the stimulus in an interval did not significantly affect subjects' duration discriminations. Similar results have been reported by Abel (1972b) and Carbotte and Kristofferson (1973).

Another possibility that could explain better performance with filled intervals than with empty intervals is based on the "misassignment" hypothesis of Kallman and his colleagues (Kallman et al., 1988; Kallman et al., 1986), which, as noted above, was inspired by their findings that forward and backward masking have equivalent effects on duration discrimination with filled auditory intervals. Equivalence of forward and backward masking suggests that both types of masking effects result from misassignment by a central timekeeper of pulses generated by an internal pacemaker. According to the process model of timing described by Church (1984), the internal clock is composed of a pacemaker, a switch, and an accumulator (which can be seen as equivalent to Kallman's central timekeeper). The pacemaker generates pulses that are switched into the accumulator. On the basis of animal studies, Church concluded that the switch can be operated in different modes, some much more complex than others. It is plausible to assume that the simplest switch mode (in which the switch is on at the onset of a signal and off at the offset of a signal) was applied to filled-interval trials in our experiments, whereas a more complex mode was applied to the empty-interval trials. If a more complex mode is more prone to error than the simplest mode, it should be the case that empty intervals are processed less accurately than filled intervals. Furthermore, Kallman and colleagues (Kallman et al., 1988; Kallman et al., 1986) have found evidence that the addition of auditory masking results in an increase in "misassignments" by the central timekeeper, and it is likely that misassignments will be more frequent in a complex switch mode than in a simple switch mode, thus causing empty intervals to suffer more from masking than filled intervals do.

A somewhat similar explanation can be derived from the framework of Robin and Royer (1987), who studied auditory temporal processing by using a flutter-fusion paradigm, in which two tones were separated by a silent interval and subjects were instructed to judge when the tone bursts fused perceptually by adjusting the duration of the first tone. Robin and Royer assumed a mutually inhibitory action between on cells (which begin to fire at stimulus onset) and off cells (which fire at stimulus offset), so that at the onset of a stimulus, the off response is inhibited. This inhibition decays, eventually allowing the off cell to fire; the latency with which this cell fires is governed by the "inverse latency-duration function," which states that the shorter the first stimulus, the longer the latency of the off cell. This inverse latency-duration function can be used to explain why our subjects performed better on filled-interval trials than on emptyinterval trials. In the empty-interval trials, the extremely brief duration of the first click $(3 \mathrm{msec}$ ) would cause a pronounced increase in the latency with which the off cell would fire. If we assume that this greater latency is accompanied by greater variability in registering click offset, and if we further assume that off cells are likely to have a lower firing rate than on cells, it should be the case that empty intervals are processed much less accurately than filled intervals. 
In conclusion, our results suggest that duration discrimination of very brief auditory intervals is based on processing at the perceptual level. These findings are in agreement with the assumption of an internal timing mechanism that is more adapted to the processing of filled intervals than to the processing of empty intervals marked by clicks, resulting in a finer temporal resolution in the case of filled intervals. This assumption is supported by additional evidence suggesting that tones are processed more efficiently than clicks. For example, measurements of interaural time-difference thresholds point to remarkable differences in sensory processing of tones and clicks. Klumpp and Eady (1956) showed that the $75 \%$ interaural timedifference threshold for $1000-\mathrm{Hz}$ tones was $11 \mu \mathrm{sec}$, but $28 \mu \mathrm{sec}$ for 1-msec clicks, indicating superior temporal resolution in the detection of differences in interaural arrival times for tones. Klumpp and Eady's results suggest differences in sensory processing of tones and clicks at low neural levels beyond cognitive control. The outcome of the present experiments done with the durationdiscrimination paradigm demonstrates that superior performance on filled intervals as opposed to empty intervals is not due to a difference in cognitive demands but to a difference in perceptual processing.

\section{REFERENCES}

ABEL, S. M. (1972a). Discrimination of temporal gaps. Journal of the Acoustical Society of America, 52, 519-524.

ABEL, S. M. (1972b). Duration discrimination of noise and tone bursts. Journal of the Acoustical Society of America, 51, 1219-1223.

Allan, L. G. (1979). The perception of time. Perception \& Psychophysics, 26, 340-354.

Allan, L. G., Kristofrerson, A. B. (1974). Psychophysical theories of duration discrimination. Perception \& Psychophysics, 16, 26-34.

Allan, L. G., \& Rousseau, R. (1977). Backward masking in judgments of duration. Perception \& Psychophysics, 21, 482-486.

Avant, L. L., Lyman, P. J., \& ANTEs, J. R. (1975). Effects of stimulus familiarity upon judged visual duration. Perception \& Psychophysics, 17, 253-262.

BADDELEY, A. D. (1981). The concept of working memory: A view of its current state and probable future development. Cognition, 10, 17-23.

BAdpeley, A. D., Hrtch, G. (1974). Working memory. In G. H. Bower (Ed.), The psychology of leaming and motivation (Vol. 8, pp. 47-89). New York: Academic Press.

BLOCK, R. A. (1974). Memory and the experience of duration in retrospect. Memory \& Cognition, 2, 153-160.

BLOCK, R. A., ReED, M. A. (1978). Remembered duration: Evidence for a contextual change hypothesis. Joumal of Experimental Psychology: Human Learning \& Memory, 4, 656-665.

Carbotte, R. M., \& Kristofferson, A. B. (1971). Discrimination of brief empty intervals (Tech. Rep. No. 21). Hamilton, Ontario, Canada: McMaster University, Department of Psychology.

Carbotte, R. M., \& Kristofferson, A. B. (1973). On energydependent cues in duration discrimination. Perception \& Psychophysics, 14, 501-505.

Church, R. M. (1984). Properties of the internal clock. In J. Gibbon \& L. G. Allan (Eds.), Timing and time perception (Annals of the New York Academy of Sciences: Vol. 423, pp. 566-582). New York: New York Academy of Sciences.
Cralg, J. C. (1973). A constant error in the perception of brief temporal intervals. Perception \& Psychophysics, 13, 99-104.

Creelman, C. D. (1962). Human discrimination of auditory duration. Journal of the Acoustical Society of America, 34, 582-593.

Deatherage, B. H., Evans, T. R. (1969). Binaural masking: Backward, forward, and simultaneous effects. Joumal of the Acoustical Sociery of America, 46, 362-371.

Evans, E. F. (1975). Cochlear nerve and cochlear nucleus. In W. D. Keidel \& W. D. Neff (Eds.), Handbook of sensory physiology: Vol. S: Pt. 2 (pp. 1-108). Berlin: Springer-Verlag.

Fortin, C., \& Rousseau, R. (1987). Time estimation as an index ofprocessing demand in memory search. Perception \& Psychophysics, 42, 377-382.

Fraisse, P. (1979). Influence de la durte du traitement de l'information sur l'estimation d'une durte d'une seconde. Anrée Psychologique, 79, 495-504

Hawkins, H. L., Presson, J. C. (1977). Masking and perceptual selectivity in auditory recognition. In S. Dornic (Ed.), Artention and performance VI (pp. 195-211). New York: Wiley.

Kallman, H. J., Beckstead, J. W., Cameron, P. A. (1988). Ipsilateral and contralateral masking of duration. Perception \& Psychophysics, 43, 31-37.

Kallman, H. J., Hirtle, S. C., Davidson, D. (1986). Recognition masking of auditory duration. Perception \& Psychophysics, 10, 45-52.

Kallman, H. J., \& Moreus, M. D. (1984). Duration perception and auditory masking. In J. Gibbon \& L. G. Allan (Eds.), Timing and time perception (Annals of the New York Academy of Sciences: Vol. 423, pp. 608-609). New York: New York Academy of Sciences.

KLUMPP, R. G., EADY, H. R. (1956). Some measurements of interaural time difference thresholds. Joumal of the Acoustical Sociery of America, 28, 859-860.

LEVITT, H. (1971). Transformed up-down methods in psychoecoustics. Journal of the Acoustical Society of America, 49, 467-477.

Massaro, D. W. (1972). Perceptual images, processing time, and perceptual units in auditory perception. Psychological Review, 79, 124-145.

Massaro, D. W. (1976). Auditory information processing. In W. K. Estes (Ed.), Handbook of learning and cognitive processes (Vol. 4, Pp. 275-320). Hillsdale, NJ: Erlbaum.

Massaro, D. W., Cohen, M. M., IDson, W. L. (1976). Recognition masking of auditory lateralization and pitch judgments. Journal of the Acoustical Society of America, 59, 434-441.

Michon, J. A. (1985). The compleat time experiencer. In J. A. Michon \& J. L. Jackson (Eds.), Time, mind, and behavior (pp. 21-52). Berlin: Springer-Verlag.

Mrtrani, L., Shekerdiski, S., Gourevitch, A., Yanev, S. (1977). Identification of short time intervals under LSD-25 and mescaline. Activitas Nervosa Superior, 19, 103-104.

Mulugan, R. M., SChiffMan, H. R. (1979). Temporal experience as a function of organization in memory. Bulletin of the Psychonomic Society, 14, 417-420.

ORNSTEIN, R. E. (1969). On the experience of time. Harmondsworth, U.K.: Penguin Books.

PoYnter, W. D., Homa, D. (1983). Duration judgment and the experience of change. Perception \& Psychophysics, 33, 548-560.

RAMMSA YER, T. (1991). Zeitdauerdiskrimination bei Blinden und NichsBlinden: Evidenz fur einen biologischen zeitmechanismus. [Temporal discrimination of the blind and the sighted: Evidence for a biological timing mechanism.]. Manuscript submitted for publication.

Rammsayer, T., Vogel, W. H., Serota, R. D., * Gottheil, E. (1989). Chronomutagenic effect of alcohol on timing mechanisms in humans. Pharmacologist, 31, 156.

Robin, D. A., RoYer, F. L. (1987). Auditory temporal processing: Two-tone flutter fusion and a model of temporal integration. Joumal of the Acoustical Society of America, 82, 1207-1211.

SHIFFRIN, R. M. (1976). Capacity limitations in information processing, attention, and memory. In W. K. Estes (Ed.), Handbook of learning and cognitive processes (Vol. 4, pp. 177-236). Hillsdale, NJ: Erlbaum. 
Thomas, E. A. C., Weaver, W. B. (1975). Cognitive processing and time perception. Perception \& Psychophysics, 17, 363-367.

Treisman, M. (1963). Temporal discrimination and the indifference interval: Implications for a model of the "internal clock." Psychological Monographs, 77, 1-31.

\section{APPENDIX \\ Word List for Experiments 2 and 3}

\section{Nouns (Experiments 2 and 3)}

Berechnung (calculation), Biene (bee), Bleistift (pencil), Durst (thirst), Eitelkeit (vanity), Flugzeug (airplane), Freiheit (freedom), Freund (friend), Gesetz (law), Gewissen (conscience), Handball (handball), Himmel (sky), Krawatte (tie), Kunst (art), Meuterei (mutiny), Nelke (carnation), Paket (package), Rettich (radish), Rucksicht (respect), Schuhmacher (shoemaker), Ses- sel (armchair), Taschentuch (handkerchief), Technik (technology), Ungeduld (impatience), Zimmer (room).

\section{Verbs (Experiment 2)}

anrufen (to call), arbeiten (to work), aufschieben (to suspend), beten (to pray), betonen (to emphasize), betteln (to beg), denken (to think), fahren (to drive), fallen (to fall), lachen (to laugh), legen (to pull), rauben (to rob), rechnen (to reckon), reinigen (to clean), riechen (to smell), segeln (to sail), spielen (to gamble), stottern (to stutter), teilen (to divide), tragen (to carry), verhandeln (to negotiate), verschlafen (to oversleep), versuchen (to try), verweigern (to refuse), zeichnen (to draw).

(Manuscript received January 11, 1991; revision accepted for publication July 31,1991 .)

\title{
Announcement
}

\author{
Third International Conference on Visual Search \\ University of Nottingham \\ August 24-27, 1992
}

\section{Call for Papers}

The Applied Vision Association, in conjunction with the Ergonomics Society and the British Machine Vision Association and Society for Pattern Recognition, is currently organizing the Third International Conference on Visual Search to be held at the University of Nottingham, U.K., August 24-27, 1992.

This is the third in a series of international conferences devoted exclusively to all aspects of visual search processing. The meeting will encompass both human and machine vision approaches. The sessions will include (but not be limited to): attention and segmentation, eye movements, computer vision, search modeling, and applied aspects of search. There will also be a workshop, and the conference proceedings will be published.

Abstracts ( 250 words maximum, indicating whether oral or poster presentation preferred) should be submitted to the Conference Secretariat at the address given below. The deadline for the receipt of abstracts is February 7, 1992, and for submission of full manuscripts, July 3, 1992.

All correspondence and inquiries about the conference should be addressed to: TICVS, Academic Radiology, University Hospital, Queens Medical Centre, Clifton Boulevard, Nottingham NG7 2UH, U.K. (Tel., 44(0)602 709442; FAX/ANSWERPHONE, 44(0)602 709140). 